\title{
openheart Rationale and design of the Coronary Microvascular Angina Cardiac Magnetic Resonance Imaging (CorCMR) diagnostic study: the CorMicA CMR sub-study
}

David Corcoran, ${ }^{1,2}$ Thomas J Ford, ${ }^{1,2}$ Li-Yueh Hsu, ${ }^{3}$ Amedeo Chiribiri, ${ }^{4}$ Vanessa Orchard, ${ }^{2}$ Kenneth Mangion, ${ }^{1,2}$ Margaret McEntegart, ${ }^{2}$ Paul Rocchiccioli, ${ }^{2}$ Stuart Watkins, ${ }^{2}$ Richard Good, ${ }^{2}$ Katriona Brooksbank, ${ }^{1}$ Sandosh Padmanabhan, ${ }^{1}$ Naveed Sattar, ${ }^{1}$ Alex McConnachie, ${ }^{5}$ Keith G Oldroyd, ${ }^{2}$ Rhian M Touyz, ${ }^{1}$ Andrew Arai, ${ }^{3}$ Colin Berry ${ }^{1,2}$

To cite: Corcoran D, Ford TJ, Hsu L-Y, et al. Rationale and design of the Coronary Microvascular Angina Cardiac Magnetic Resonance Imaging (CorCMR) diagnostic study: the CorMicA CMR sub-study. Open Heart 2018;5:e000924.

doi:10.1136/

openhrt-2018-000924

Received 21 August 2018 Revised 24 September 2018 Accepted 12 November 2018

Check for updates

(c) Author(s) (or their employer(s)) 2018. Re-use permitted under CC BY. Published by BMJ.

For numbered affiliations see end of article.

Correspondence to Dr David Corcoran; dcorcoran@ nhs.net

\section{ABSTRACT}

Introduction Angina with no obstructive coronary artery disease (ANOCA) is a common syndrome with unmet clinical needs. Microvascular and vasospastic angina are relevant but may not be diagnosed without measuring coronary vascular function. The relationship between cardiovascular magnetic resonance (CMR)-derived myocardial blood flow (MBF) and reference invasive coronary function tests is uncertain. We hypothesise that multiparametric CMR assessment will be clinically useful in the ANOCA diagnostic pathway.

Methods/analysis The Stratified Medical Therapy Using Invasive Coronary Function Testing In Angina (CorMicA) trial is a prospective, blinded, randomised, sham-controlled study comparing two management approaches in patients with ANOCA. We aim to recruit consecutive patients with stable angina undergoing elective invasive coronary angiography. Eligible patients with ANOCA ( $n=150)$ will be randomised to invasive coronary artery function-guided diagnosis and treatment (intervention group) or not (control group). Based on these test results, patients will be stratified into disease endotypes: microvascular angina, vasospastic angina, mixed microvascular/vasospastic angina, obstructive epicardial coronary artery disease and non-cardiac chest pain. After randomisation in CorMicA, subjects will be invited to participate in the Coronary Microvascular Angina Cardiac Magnetic Resonance Imaging (CorCMR) substudy. Patients will undergo multiparametric CMR and have assessments of MBF (using a novel pixel-wise fully quantitative method), left ventricular function and mass, and tissue characterisation (T1 mapping and late gadolinium enhancement imaging). Abnormalities of myocardial perfusion and associations between MBF and invasive coronary artery function tests will be assessed. The CorCMR substudy represents the largest cohort of ANOCA patients with paired multiparametric CMR and comprehensive invasive coronary vascular function tests.

\section{Key questions}

What is already known about this subject?

- Angina with no obstructive coronary artery disease (ANOCA) is a common syndrome with unmet clinical needs.

- A significant proportion of these patients may suffer from microvascular and vasospastic angina.

- Diagnosis in this patients may be challending, are there are uncertain associations between the results of reference invasive diagnostic tests and the non-invasive ischaemia test results.

What does this study add?

- Novel CMR methods for measuring myocardia blood flow have not been validated in patients with ANOCA and underlying microvascular and vasospastic angina.

- The CorCMR substudy represents the largest cohort of ANOCA patients with paired multiparametric CMR and comprehensive invasive coronary vascular function tests. CorCMR will provide information on the diagnostic value of quantitative pixel-wise mapping of myocardial perfusion in patients with ANOCA.

How might this impact on clinical practice?

- In contrast to the reference standard invasive tests of coronary artery function, non-invasive imaging is safer and more acceptable to patients.

- The CorCMR sub-study presents a unique opportunity to assess and validate the diagnostic accuracy of fully-quantitative stress perfusion CMR in patients with ANOCA and comprehensive invasive coronary artery function testing.

Ethics/dissemination The CorMicA trial and CorCMR substudy have UK REC approval (ref.16/WS/0192). Trial registration number NCT03193294. 


\section{INTRODUCTION}

\section{Angina and stable coronary syndromes (SCSs)}

Ischaemic heart disease is the leading cause of mortality standardised by age and sex. ${ }^{1}$ In clinical practice, the diagnostic management of patients with angina pectoris focuses on the detection of obstructive epicardial coronary artery disease $(\mathrm{CAD}){ }^{2}$ In this stenosis-centred concept of myocardial ischaemia, angina is synonymous with obstructive CAD. ${ }^{3}$ There are well established treatment options for patients with epicardial CAD, namely optimal medical therapy and myocardial revascularisation by either percutaneous coronary intervention or coronary artery bypass grafting. ${ }^{3}$ However, the paradigm of angina pectoris resulting from obstructive epicardial CAD fails to account for the approximately one-third of patients who suffer from angina in whom obstructive CAD is excluded. ${ }^{4}$

Patients with angina and no obstructive coronary artery disease (ANOCA) present a diagnostic conundrum. ${ }^{5}$ The management of these patients is varied, and most patients fail to have a diagnosis made for the cause of their symptoms, receive no further diagnostic work-up, and have no therapeutic intervention. The underlying aetiology of chest pain symptoms and a 'negative' coronary angiogram is heterogeneous. However, a significant number of patients may have a disorder of coronary vascular function due to abnormal microvascular resistance or vasodilator capacity (coronary microvascular dysfunction (CMD)) or abnormal endothelial function (vasospastic disease).

The underlying pathogenesis in patients with ANOCA is unclear, as specific disease endotypes are not routinely tested for. CMD may result from coronary structural abnormalities, whereby decreased capillary luminal size and number result in increased microvascular resistance to myocardial blood flow (MBF) and reduced vasodilatory capacity. ${ }^{6}$ Functional abnormalities of the coronary epicardial vessels and microvasculature may result in either abnormal vasoconstriction or impaired vasodilatation, and these abnormalities may be secondary to either endothelium-dependent or endothelium-independent mechanisms. ${ }^{7}$

Abnormalities of coronary vascular function portend a worse prognosis in patients with both obstructive epicardial CAD and ANOCA..$^{8-12}$ Therapeutic interventions are lacking in patients with ANOCA, and historical therapeutic studies have been performed in heterogeneous patient cohorts due to a lack of diagnostic tests to appropriately define endotypes of disease. ${ }^{13-15}$ There is a missing link between the use of diagnostic tests of coronary artery function, therapeutic agents with proven efficacy and health outcomes of patients with angina secondary to disorders of coronary vascular function. The term stable coronary

syndrome (SCS) has been proposed to increase physician awareness of these conditions. ${ }^{5}$ CMD and vasospastic disease may result in ANOCA and myocardial ischaemia, and are recognised as a condition of unmet clinical need. ${ }^{16}$ The Coronary Vasomotion Disorders International Study Group (COVADIS) working group have proposed diagnostic criteria for disease endotypes in patients with ANOCA. ${ }^{17}$ COVADIS recommend a comprehensive testing strategy incorporating tests of coronary pressure, flow, resistance and endothelial function, in addition to the assessment of objective evidence of myocardial ischaemia. ${ }^{17}$

\section{Diagnostic testing in patients with ANOCA}

Diagnosis of coronary microvascular and vasomotor dysfunction is challenging due to the heterogeneity of underlying disease mechanisms, the potentially patchy distribution of disease throughout the myocardium, and limited spatial resolution of existing diagnostic tests. ${ }^{18} 19$ There is no available in vivo technique for imaging the coronary microcirculation, and anatomical tests are fundamentally limited by their spatial resolution and the small size of the coronary microvasculature. Therefore, the diagnosis of microvascular angina and vasospastic angina is predominantly made with functional tests.

There is no accepted guideline-directed diagnostic algorithm for coronary vascular dysfunction in routine clinical practice. ${ }^{17} 20$ Invasive coronary angiography combined with adjunctive tests of coronary artery function represents the reference diagnostic approach for disorders of coronary vascular function. In contrast, non-invasive imaging involves less discomfort for patients, is safer than invasive procedures and is generally less expensive and more widely available. Recent developments with cardiovascular magnetic resonance (CMR) imaging now enable measurement of MBF with high spatial and temporal resolution. ${ }^{21}$ In addition, CMR permits the reference standard non-invasive assessment of left ventricular (LV) function and myocardial tissue characterisation. ${ }^{22}$

\section{Non-invasive ischaemia testing in ANOCA}

The available non-invasive ischaemia tests were all validated for the detection of obstructive epicardial CAD. There has been a low yield of inducible myocardial ischaemia in ANOCA patients with traditional non-invasive ischaemia tests (e.g. exercise ECG testing, myocardial perfusion scintigraphy and stress echocardiography). ${ }^{23}$ However, non-invasive methods (namely stress perfusion positron emission tomography (PET) and CMR which image earlier in the ischaemic cascade and have greater spatial resolution) may provide new insights into the burden of myocardial ischaemia in patients with ANOCA. PET is the most studied modality for the assessment of myocardial perfusion and is considered to provide the reference standard assessment of MBF, but in real-world clinical practice, availability is limited due to cost. Conversely, CMR is more widely available and use is increasing. ${ }^{24} 25$

Coronary microvascular disease may be revealed by a deficit in MBF during stress CMR. The spatial distribution of this abnormality typically involves the subendocardium, 
which is the location of the microvascular plexus. ${ }^{26}$ In contrast, vasospastic angina occurs due to spontaneous spasm of the epicardial and microvascular vasculature. Vasospastic angina may not be detected by conventional stress testing that routinely use adenosine (an endothelial-independent vasodilator). The available data are conflicting on the role of perfusion CMR in patients with ANOCA ${ }^{26-28}$ In contrast to qualitative analysis, semiquantitative perfusion methods have been investigated, and reduced myocardial perfusion reserve index (MPRi) has been found in patients with ANOCA. ${ }^{29}$ The incremental diagnostic value of fully quantitative perfusion CMR in patients with ANOCA is unknown.

\section{Associations between invasive and non-invasive diagnostic tests in patients with ANOCA}

There are uncertain associations between the results of invasive diagnostic tests and the non-invasive ischaemia test results in patients with ANOCA. There is no accepted objective diagnostic threshold for the diagnosis of coronary vascular dysfunction (either abnormal myocardial flow or microvascular resistance), with either PET or perfusion CMR. Recent studies have investigated the relationships between invasive tests of coronary artery function and semiquantitative perfusion CMR analysis. ${ }^{30}{ }^{31}$ Importantly, these patients have not undergone comprehensive coronary vascular function testing and in general moderate correlations were demonstrated (MPRi and index of microcirculatory resistance (IMR): $r=-0.67$; MPRi and coronary flow reserve (CFR): $r=0.41) .{ }^{30}$

\section{The Stratified Medical Therapy Using Invasive Coronary Function Testing In Angina (CorMicA) trial}

The Stratified Medical Therapy Using Invasive Coronary Function Testing In Angina (CorMicA) clinical trial is a proof-of-concept, prospective, blinded, randomised, sham-controlled study comparing two management approaches to the clinical problem of patients with ANOCA. ${ }^{32}$ CorMicA tests the hypothesis that stratified medicine guided by invasive coronary artery function testing (interventional diagnostic procedure (IDP)) in patients with ANOCA will facilitate diagnosis of the underlying disease endotype, direct therapeutic interventions aligned to the endotype and result in improved angina and well-being. ${ }^{32}$ Patients undergoing elective invasive coronary angiography for investigation of angina at two UK centres will be screened. Eligible patients with ANOCA $(n=150)$ will be immediately randomised 1:1 to either coronary vascular function-guided diagnosis and treatment (intervention group/IDP disclosed) or not (control group/IDP sham procedure, results not disclosed) (figure 1).

The IDP consists of coronary artery function testing using a dual pressure-sensitive and temperature-sensitive guidewire and adenosine followed by intracoronary acetylcholine provocation testing. Assessment of microvascular resistance (IMR), microvascular vasodilatory capacity (resistance reserve ratio (RRR)), epicardial

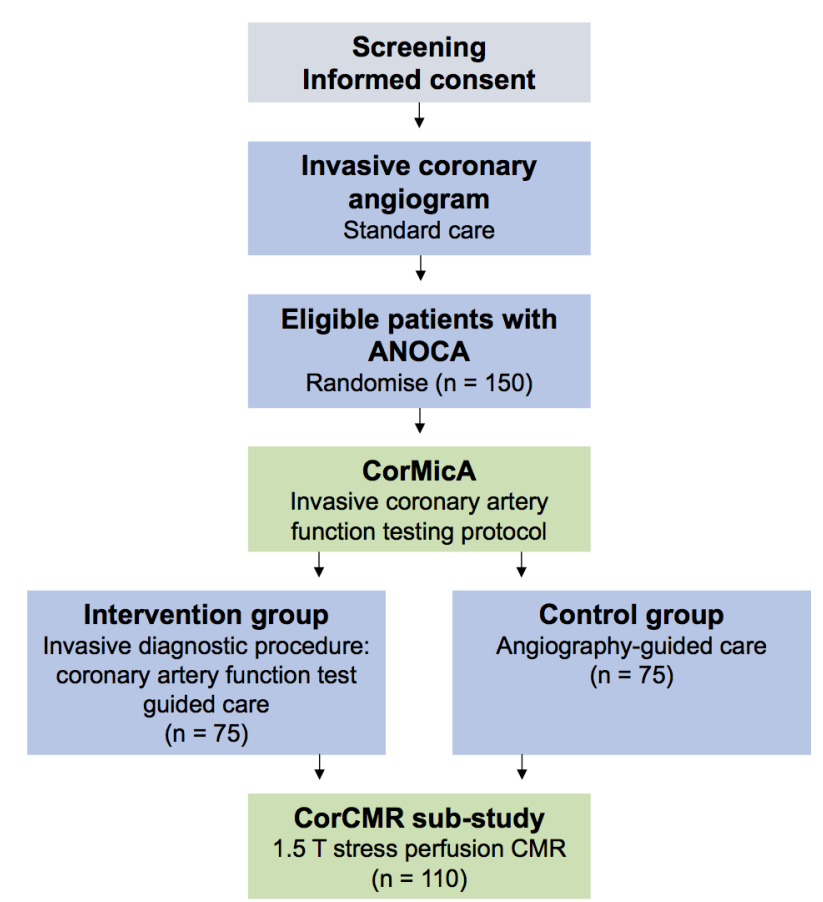

Figure 1 CorCMR substudy flow diagram. ANOCA, angina with no obstructive coronary artery disease; CMR, cardiovascular magnetic resonance; CorCMR, Coronary Microvascular Angina Cardiac MRI; CorMicA, coronary microvascular angina.

and microvascular vasodilatory capacity (CFR), endothelial function (acetylcholine provocation testing) and epicardial CAD (fractional flow reserve (FFR)) will be performed. Following these invasive tests of coronary artery function, patients will be classified into the following ANOCA disease endotypes (table 1): (1) microvascular angina; (2) vasospastic angina; (3) mixed microvascular angina and vasospastic angina; (4) obstructive epicardial CAD; and (5) non-cardiac chest pain. The disease endotypes are aligned with the COVADIS working group definitions. ${ }^{17}$

Patients in the control group will receive standard care based on the interpretation of the invasive coronary angiogram alone. Patients in the intervention group will have care based on the disease endotypes disclosed by invasive tests of artery function, and pharmacotherapy linked to the underlying disease endotype will be commenced.$^{32}$ In each case, the diagnosis (endotype) will be assessed by the attending cardiologist before and after the coronary angiogram. In the intervention group, the diagnosis is re-evaluated after disclosure of the coronary function test results at the end of the invasive procedure. The primary outcome is the mean difference in the within-subject change in Seattle Angina Questionnaire score between the groups at 6 months from baseline. A prespecified substudy of the CorMicA trial investigated the frequency of peripheral microvascular dysfunction in patients with ANOCA. $^{33}$ 


\begin{tabular}{|c|c|c|}
\hline Disease endotype & Mechanism & Invasive diagnostic test \\
\hline \multirow[t]{4}{*}{ Microvascular angina } & $\uparrow$ Microvascular resistance & IMR $\geq 25$ \\
\hline & $\downarrow$ Coronary vasorelaxation & CFR $<2.0$ \\
\hline & $\downarrow$ Microvascular vasodilator capacity & $\mathrm{RRR}<2.0$ \\
\hline & Microvascular spasm & $\begin{array}{l}\text { ACh testing: angina, ischaemic ST segment deviation, epicardial coronary } \\
\text { vasoconstriction }<90 \% \text {. }\end{array}$ \\
\hline Vasospastic angina & Epicardial spasm & $\begin{array}{l}\text { ACh testing: angina, ischaemic ST segment deviation, }>90 \% \text { epicardial coronary } \\
\text { vasoconstriction. }\end{array}$ \\
\hline $\begin{array}{l}\text { Mixed microvascular and vasospastic } \\
\text { angina }\end{array}$ & CMD and epicardial vasospasm & $\begin{array}{l}\text { Epicardial vasospasm and either } \uparrow \text { microvascular resistance, } \downarrow \text { coronary } \\
\text { vasorelaxation or } \downarrow \text { microvascular vasodilator capacity. }\end{array}$ \\
\hline $\begin{array}{l}\text { Obstructive epicardial } \\
\text { CAD }\end{array}$ & Epicardial stenosis & $>50 \%$ lesion by diameter stenosis in epicardial artery $>2.5 \mathrm{~mm}$ or $\mathrm{FFR} \leq 0.80$. \\
\hline Non-cardiac pain & Nil & $\begin{array}{l}\text { Exclusion of epicardial (FFR }>0.8 \text { ), microvascular (CFR }>2.0 \text {, IMR }<25 \text {, RRR }>2.0 \text { ) } \\
\text { vasospasm (normal ACh response). }\end{array}$ \\
\hline
\end{tabular}

ACh, acetylcholine; ANOCA, angina with no obstructive coronary artery disease; CAD, coronary artery disease; CFR, coronary flow reserve; CMD, coronary microvascular dysfunction; FFR, fractional flow reserve; IMR, index of microcirculatory resistance; RRR, relative resistance ratio.

\section{Coronary Microvascular Angina Cardiac Magnetic Resonance Imaging (CorCMR) sub-study}

Novel CMR methods for measuring MBF have not been validated in patients with ANOCA and underlying microvascular and vasospastic angina. The CorCMR substudy of the CorMicA clinical trial presents a unique opportunity to assess and validate the diagnostic accuracy of fully quantitative stress perfusion CMR in patients with ANOCA and comprehensive invasive coronary artery function testing.

\section{Hypothesis}

We hypothesise that abnormal myocardial perfusion, as revealed by a novel fully quantitative pixel-wise CMR perfusion sequence, will be prevalent in a contemporary UK cohort of patients presenting with ANOCA and that multiparametric CMR imaging will be clinically useful in the diagnostic pathway of patients with ANOCA.

\section{Aims}

We aim to assess the diagnostic validity of quantitative stress perfusion CMR in patients with ANOCA and specific disease endotypes of coronary vascular dysfunction. Our specific aims are to assess:

1. The proportion of ANOCA patients with abnormal myocardial perfusion. There is no accepted threshold for abnormal MPR. Thresholds for reduced CFR of 1.5-2.6 have been described,however $<2.0$ is commonly used. ${ }^{10}{ }^{34-38}$ We will therefore assess the concordance of patient classification using this MPR $<2.0$ threshold against endotypes determined by invasive testing. Second, we will assess the MPR value with the highest area under the curve (AUC) for classification of microvascular angina based on the invasive tests (reference classification). Third, we will assess the proportion of ANOCA endotypes with other abnormal perfusion metrics (reduced stress MBF, abnormal endocardial:epicardial MBF ratio and abnormal myocardial dyssynchrony index). ${ }^{39}$

2. The diagnostic accuracy of the perfusion CMR metrics for abnormal invasive tests of coronary artery function (IMR > 25, CFR <2.0 and RRR <2.0) and the associations between specific disease endotypes and myocardial perfusion in patients with ANOCA. We also aim to assess the correlation between abnormal CMR-derived perfusion and invasive tests of coronary artery function, of qualitative versus quantitative perfusion methods.

3. The proportion of patients with ANOCA and abnormal myocardial tissue characterisation derived from CMR (as revealed by T1 parametric mapping and late gadolinium enhancement (LGE) imaging) and its association with abnormal myocardial perfusion.

4. The associations between baseline patient characteristics and abnormal myocardial perfusion.

5 . The proportion of patients with a change in diagnosis based on quantitative CMR findings, as compared with the initial diagnosis by the attending cardiologist based on the coronary angiogram.

\section{METHODS AND ANALYSIS \\ Study design}

Prespecified substudy of the CorMicA stratified medical therapy clinical trial.

\section{Setting}

Patients referred from 14 acute hospitals to two large regional UK hospitals (Golden Jubilee National Hospital and Hairmyres Hospital) providing invasive care to all patients in the West of Scotland (population 2.5 million). All CMR studies will be performed at the Golden Jubilee National Hospital. 


\section{Participants}

Consecutive outpatients undergoing clinically indicated elective diagnostic angiography for investigation of suspected angina will be screened and invited to participate in the CorMicA trial. Informed consent is obtained before the invasive coronary angiogram. A minimum of 400 consecutive patients undergoing elective invasive coronary angiography is expected to be screened to enrol 150 subjects with ANOCA within 24 months. Consenting patients who are not randomised (e.g. demonstrated to have obstructive epicardial disease or logistical reasons) will enter a registry. Only patients randomised in the CorMicA trial (both in the intervention and control groups) are eligible to participate in the CorCMR substudy.

\section{Inclusion criteria}

Age $\geq 18$ years, a clinically indicated plan for invasive coronary angiography and symptoms of angina (according to the Rose angina questionnaire).

\section{Exclusion criteria}

A non-coronary indication for invasive angiography (e.g. valvular heart disease and cardiomyopathy), obstructive epicardial CAD disease evident in a main coronary artery (diameter $>2.5 \mathrm{~mm}$ ) (defined as diameter stenosis $>50 \%$ or FFR $\leq 0.80$ ) and contraindication to contrast-enhanced CMR (glomerular filtration rate $<30 \mathrm{~mL} / \mathrm{min}$, CMR unsafe devices).

\section{CMR protocol and analysis}

Patients will undergo perfusion CMR within 42 days of the CorMicA trial invasive coronary artery function testing. CMR studies will be performed at 1.5 Tesla (Siemens MAGNETOM Avanto, Erlangen, Germany), and patients will undergo a standardised CMR protocol. All patients will be asked to abstain from caffeine-containing beverages or foodstuffs for 24 hours and vasoactive medications for 48 hours prior to the CMR examination. All scan acquisitions will be spatially coregistered. All CMR analyses will be performed by analysts blinded to the invasive coronary artery function test results. The standardised CMR protocol is demonstrated in figure 2.

\section{Myocardial perfusion}

Stress and rest first-pass perfusion imaging will be performed using an echo planar imaging dual-sequence investigational perfusion method, which consists of a low resolution arterial input function image, followed by three short axis (base, mid and apex) myocardial images during each R-R interval. ${ }^{40-42}$ Vasodilator stress will be achieved with adenosine infusion $140-210 \mu \mathrm{g} / \mathrm{kg} / \mathrm{min}$ for $3 \mathrm{~min}$. Resting first-pass perfusion will be performed at least 10 min later.

The raw stress and rest perfusion images will be qualitatively assessed for inducible or fixed perfusion defects. Perfusion defects will be reported on a segmental basis according to the American Heart Association 16-segment model. ${ }^{43}$ Dark banding artefact will be adjudicated based on standardised criteria. ${ }^{22}$

Pixel-wise perfusion maps will be generated and analysed to derive fully quantitative MBF estimates on a pixelwise basis in $\mathrm{mL} / \mathrm{g} / \mathrm{min}$ of myocardium. The pixel-wise perfusion method uses a series of automated postprocessing steps on the raw Digital Imaging and Communications in Medicine images to generate fully quantitative pixel maps. ${ }^{21}$ The pixel-wise time-signal intensity curves will then be quantified using model-constrained Fermi deconvolution. ${ }^{44-46}$

The myocardial perfusion dyssynchrony index is a novel perfusion metric that assesses temporal differences in the distribution of gadolinium-based contrast media myocardial wash-in. This index has the potential to increase the diagnostic sensitivity and specificity of perfusion CMR for abnormalities of myocardial perfusion in patients
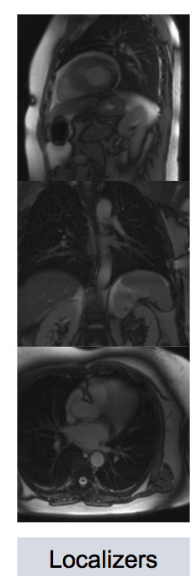

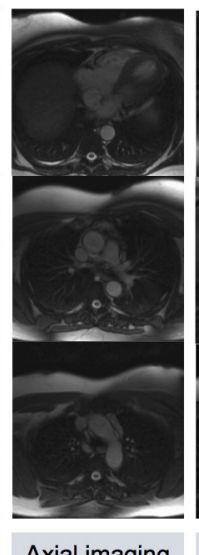

Axial imaging
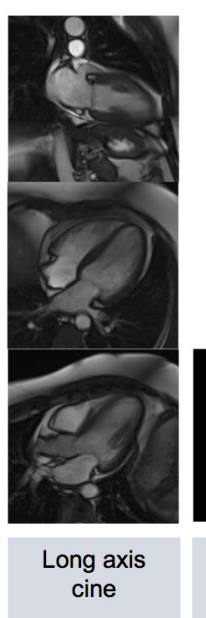
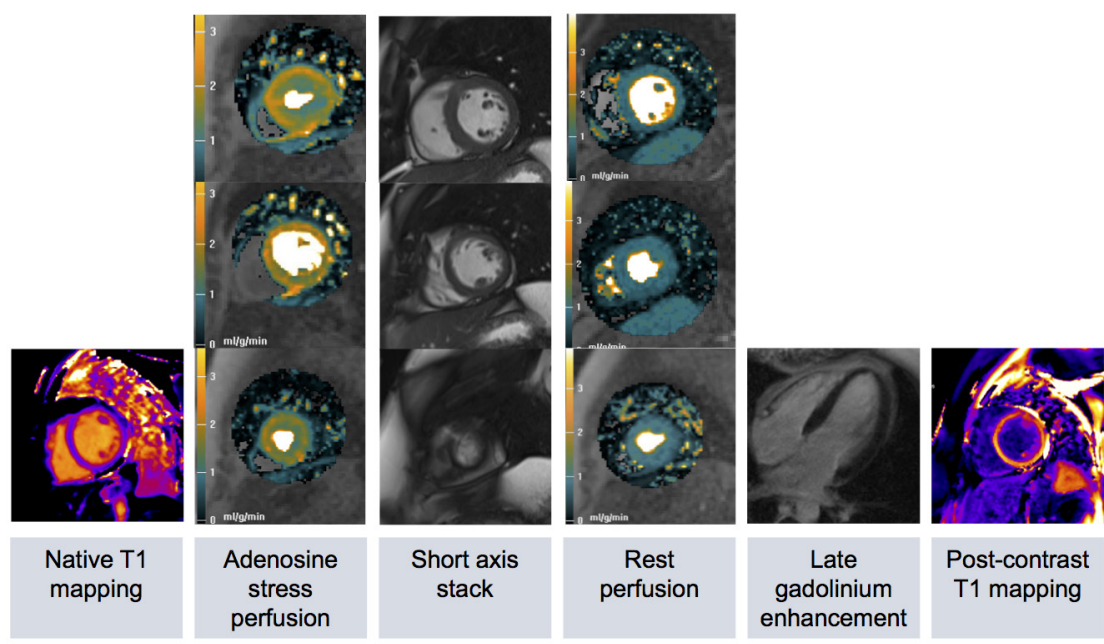

60 mins

Figure 2 CorCMR multiparametric imaging protocol. CorCMR, Coronary Microvascular Angina Cardiac MRI. 
Box 2. CorCMR substudy secondary outcomes and analyses

LV volumes, function and mass

- LVEF

- LVEDV

- LV EDV index

- LVESV

- LV ESV index

- LV mass

- LV mass index

- LV CO

- LV CO index

Atrial area

- Left atrial area $\left(\mathrm{cm}^{2}\right)$

- Left atria dilated $(\mathrm{Y} / \mathrm{N})$

- Right atrial area $\left(\mathrm{cm}^{2}\right)$

- Right atria dilated $(\mathrm{Y} / \mathrm{N})$

Qualitative perfusion analysis

- Abnormal perfusion $(\mathrm{Y} / \mathrm{N})$

- Number of abnormal segments (n)

- Transmurality of perfusion defects (\%)

- Pattern (epicardial, microvascular, mixed and equivocal)

Quantitative perfusion analysis

- Global MPR $<2.0(\mathrm{Y} / \mathrm{N})$

- Segmental/AHA MPR

- Global stress and rest MBF

- Segmental/AHA territory stress and rest MBF

- Global stress endocardial:epicardial MPR ratio

- Segmental/AHA territory endocardial:epicardial MPR ratio

Perfusion dyssynchrony analysis

- Myocardial perfusion dyssynchrony index (variance and coefficient of variation of the time to maximum signal intensity upslope and time to peak myocardial signal intensity enhancement)

\section{Adenosine vasodilator stress response}

- Splenic switch-off (Y/N)

- HR and BPchange

- Rate-pressure product at rest and stress

Late gadolinium enhancement imaging

- Abnormal LGE $(\mathrm{Y} / \mathrm{N})$.

- Number of affected AHA segments ( $n$ )

- Pattern of abnormal LGE (ischaemic and non-ischaemic)

- Myocardial infarct scar burden (transmurality, infarct mass, infarct mass as percentage LV mass)

\section{Native T1 and ECV mapping}

- Native T1 (global and midseptal values)

- ECV (global and midseptal values)

Myocardial strain

- Feature-tracking and DENSE (two methods)

- Longitudinal strain (global and by AHA segment)

- Circumferential strain (global and by AHA segment)

- Radial strain (global and by AHA segment)

Incidental findings

- Present (Y/N)

- Clinically significant $(\mathrm{Y} / \mathrm{N})$

\section{Box 2 Continued}

Associations of abnormal perfusion with baseline characteristics

- Sex, age and traditional CAD risk factors

- CAD risk scores (JBS3 and ASSIGN)

- Gensini score of epicardial plaque burden

Associations of abnormal perfusion with invasive coronary artery function testing data

- Association of myocardial perfusion with IMR, CFR, RRR and endothelial function testing (continuous and binary)

- Subset of patients with multivessel invasive coronary artery function measurements

- Association with clinical diagnosis (ANOCA disease endotype)

Associations of abnormal perfusion with baseline CMR data

- LV volumes and mass

- Myocardial strain

- Native T1 relaxation time and ECV

- Presence of LGE

Diagnostic accuracy

- Diagnostic accuracy of quantitative perfusion CMR to detect abnormal IMR, CFR, RRR and endothelial dysfunction

AHA, American Heart Association; ANOCA, angina with no obstructive coronary artery disease; $\mathrm{BP}$, blood pressure; CFR, coronary flow reserve; CAD, coronary artery disease; $\mathrm{CO}$, cardiac output; CorCMR, Coronary Microvascular Angina Cardiac MRI; EF, ejection fraction; EDV, end-diastolic volume; ESV, end-systolic volume; ECV, extracellular volume; HR, heart rate; IMR, index of microcirculatory resistance; LV, left ventricular; LGE, late gadolinium enhancement; MBF, myocardial blood flow; MPR, myocardial perfusion reserve; RRR, relative resistance ratio,

with ANOCA. ${ }^{39}$ Global perfusion dyssynchrony indices will be measured as both variance $\left[\mathrm{s}^{2}\right]$ and percentage coefficient of variation of time to maximum signal intensity upslope and time to peak myocardial signal intensity enhancement.

\section{Extracardiac anatomy and LV volumes, function and mass}

Fast gradient echo images in the axial, coronal and sagittal planes will be qualitatively assessed for extra cardiac anatomy and pathology and clinically relevant incidental findings.

Steady-state free procession 'cine' imaging using a trueFISP sequence (multislice single-shot breath-hold true fast imaging) will be performed in the three longaxis and short-axis planes for assessment of LV volumes, function and mass.

\section{Myocardial tissue characterisation}

Native T1 mapping will be performed using a modified look-locker inversion recovery investigational prototype sequence. Images will be obtained in three shortaxis images (base, mid and apex). T1 mapping will be performed pre- and post-gadolinium contrast to assess the myocardial native $\mathrm{T} 1$ relaxation time and estimate the myocardial extracellular volume (ECV) in both the 
mid-septum and globally. ${ }^{47}$ For the calculation of ECV, blood haematocrit will be measured at baseline on enrolment into the CorMicA trial. ECV mapping analysis will be performed offline using proprietary software.

LGE imaging will be performed using a segmented phase-sensitive inversion recovery turbo fast low-angle shot imaging sequence. ${ }^{48}$ Images will be obtained in the three long-axis planes and short-axis images covering the entire LV myocardium. The pattern and burden of hyperenhancement will be qualitatively and quantitatively assessed.

\section{Primary and secondary outcomes}

\section{Primary outcome}

Abnormal myocardial perfusion reserve (global MPR $<2.0)$.

\section{Secondary outcomes}

A summary of the secondary outcomes is shown in Box 1 .

\section{Statistical analyses}

The CorMicA trial has a comprehensive statistical analysis plan that governs all statistical aspects of the study authored by the trial statistician. The statistical analysis plan includes the prespecified CorCMR substudy that is designed to assess for associations between CMR measures and invasive measures and endotypes (reference dataset). Continuous outcomes will be analysed using linear regression with adjustment for baseline levels where available. Where continuous data are clearly not normally distributed, standard transformations will be applied to achieve approximate normality prior to analysis. Appropriate alternative regression methods will be applied to other types of data (eg, logistic regression for binary outcomes).

\section{Sample size calculation}

The primary outcome is the proportion of patients with an MPR $<2.0$. The proportion of patients with microvascular angina defined by invasive endotyping will be assessed. We will further assess the MPR ratio with the highest AUC for microvascular angina classified invasively.

Considering the correlation between CFR measured invasively and MPR measured non-invasively within the common territory of a major epicardial coronary artery, then a sample size of 110 subjects would enable a minimum clinically significant correlation of 0.3 to be detected with $90 \%$ power at a $5 \%$ significance level. If only 60 subjects have available data, then $80 \%$ power would be available to detect a correlation of 0.37 at the $5 \%$ level.

\section{DISCUSSION}

The British Heart Foundation CorMicA trial will assess a routine stratified medicine strategy in a large cohort of prospectively enrolled patients with ANOCA. The prespecified CorCMR substudy will involve comprehensive invasive tests of coronary artery function paired with multiparametric perfusion CMR studies. The analysis will provide information on the diagnostic value of quantitative pixel-wise mapping of myocardial perfusion in this population.

Contemporary guidelines recommend functional testing, including with CMR, to assess for myocardial ischaemia in patients in whom multidetector CT coronary angiography has shown $\mathrm{CAD}$ of uncertain functional significance or is non-diagnostic. ${ }^{49}$ Increasingly, patients are referred to the catheter laboratory based on the results of anatomical imaging using CT coronary angiography or with no prior tests based on symptoms and a high likelihood of CAD. ${ }^{49} 50$ The CorCMR substudy in CorMicA aims to inform this gap.

The CorCMR substudy will be performed on a 1.5 Tesla MRI scanner. In comparison, 3.0 Tesla imaging permits improved signal-to-noise ratio and provides higher in-plane spatial resolution perfusion imaging. ${ }^{5152}$ However, CMR imaging in the NHS is most widely performed at 1.5 Tesla, hence the results from CorCMR are clinically relevant and transferable.

\section{Literature review}

Patients with confirmed microvascular or vasospastic angina have a precise diagnosis of the underlying disease endotype, and pharmacotherapy may be commenced as appropriate. Conversely, patients with normal invasive coronary artery function tests may have antianginal therapy appropriately discontinued, and alternative causes of chest pain investigated. In comparison with invasive tests of coronary artery function, non-invasive CMR imaging may be more attractive to patients, but at present, the role of CMR in the diagnostic work-up of patients is uncertain. CorCMR will provide data on the role of CMR in the diagnosis of patients with ANOCA. Traditional non-invasive ischaemia testing in patients with ANOCA has provided mixed results. Panza et $a l^{23}$ investigated the yield of non-invasive ischaemia testing in 70 patients with ANOCA. Patients underwent exercise ECG testing, radionuclide angiography, myocardial perfusion scintigraphy and dobutamine stress echocardiography. Abnormal test results were detected in $31 \%, 16 \%, 18 \%$ and $0 \%$ respectively, with no concordance between the test results. This led to the conclusion that patients with ANOCA did not have inducible myocardial ischaemia as a cause for their symptoms. However, there is evidence that perfusion CMR may detect abnormalities of MBF in patients with ANOCA. Panting $e t a l^{26}$ first described the use of adenosine stress perfusion CMR in patients with ANOCA. Twenty patients with syndrome $\mathrm{X}$ and 10 control subjects were included, and subendocardial hypoperfusion was demonstrated in patients with syndrome $\mathrm{X}$ using a semiquantitative perfusion metric. A subset of 118 women with ANOCA enrolled in the National Heart, Lung and Blood Institute-sponsored multicentre Women's Ischemic Syndrome Evaluation (WISE) study underwent stress perfusion CMR and invasive microvascular function testing with Doppler wire-derived CFR and 
endothelial function testing with intracoronary acetylcholine. ${ }^{29}$ Stress perfusion CMR was also performed in 21 asymptomatic control subjects. Reduced global MPRi was found in patients with ANOCA compared with controls (1.79 vs $2.23, \mathrm{p}<0.0001)$, and a lower MPRi was predictive of $\geq 1$ abnormal invasive coronary vascular function metric $(\mathrm{OR}=0.78, \mathrm{p}<0.0001)$. An MPRi threshold of 1.84 predicted an abnormal invasive coronary function metric with sensitivity of $73 \%$ and specificity of $74 \%$. Qualitative analysis of the perfusion studies found no significant differences between the study and control groups (summed segments with abnormal perfusion 6.66 vs. $4.45, \mathrm{p}=0.09$ ), suggesting that quantitative perfusion analyses may have greater sensitivity for abnormal perfusion in patients with ANOCA.

CorCMR will inform the nascent evidence on the presence and magnitude of associations between invasive and non-invasive assessments of coronary vascular function. Liu et al compared semiquantitative perfusion CMR against IMR measurement in 50 patients with ANOCA and 20 age-matched healthy control subjects. ${ }^{30}$ In a ROC analysis, an MPRi threshold of 1.4 was optimal for the detection of myocardium with inducible ischaemia in patients with obstructive epicardial CAD (FFR $\leq 0.8$ ) and in patients with ANOCA. Similarly, Williams et $a l^{\beta 1}$ performed semiquantitative perfusion CMR and measured thermodilution and Doppler flow wire-derived metrics of microvascular resistance (IMR and hyperaemic microvascular resistance (hMR), respectively) in a heterogeneous cohort of 54 patients (44 with acute myocardial infarction and 10 with stable angina). Microvascular function testing was performed in unobstructed epicardial coronary arteries (FFR $>0.8$ ). MPRi correlated with hMR $(\mathrm{r}=0.58, \mathrm{p}<0.001)$ but not IMR $(\mathrm{r}=-0.27, \mathrm{p}=0.15)$. These comparisons are between $\mathrm{a}$ non-invasive metric of vasodilatory capacity (MPRi) and an invasive metric of microvascular resistance (IMR and hMR), which may more specifically reflect fixed structural changes in the microcirculation (rather than vasodilator capacity).

CMR permits the reference-standard non-invasive assessment of myocardial tissue characterisation. CorCMR will provide data on diffuse interstitial fibrosis, ECV and myocardial scar. The role of T1 mapping and ECV analysis in patients with ANOCA is uncertain. In a substudy of the iPOWER natural history study, 54 women with ANOCA underwent native T1 mapping and ECV analysis, PET-derived MBF measurement, and transthoracic Doppler echocardiography CFR assessment. ${ }^{53}$ There was no correlation found between abnormal coronary vascular function as revealed by PET or echocardiography-derived CFR, and myocardial native T1 or ECV. Occult myocardial infarction in patients with ANOCA may be clinically relevant. In a substudy of the WISE cohort, Wei et a $\breve{l}^{\tilde{4}}$ performed CMR imaging in 340 female patients with ANOCA. A total of 26 patients (8\%) had hyperenhancement on LGE imaging, with 18 patients having evidence of occult myocardial infarction (mean scar size $5.1 \mathrm{~g})$, and 8 patients had a non-ischaemic pattern (mean scar size $8.9 \mathrm{~g}$ ).

\section{ETHICS AND DISSEMINATION}

Progress in the trial will be monitored by the trial anager (KB) and sponsor. The study will be subject to internal and external audit that is routinely coordinated by the sponsor. An annual report will be submitted to the Research Ethics Committee on a 12-month basis. The flow diagram illustrates conservative estimates of patient enrolment and activity on a single site. The study will follow Standards for Reporting of Diagnostic Accuracy (STARD) (http://www.equator-network.org/reportingguidelines/stard/) and Consolidated Standards of Reporting Trials (http://www.consort-statement.org/) guidelines.

The CorCMR data will be presented at conferences and/or published in peer-reviewed journals.

\section{Author affiliations}

${ }^{1}$ British Heart Foundation Glasgow Cardiovascular Research Centre, University of Glasgow, Glasgow, UK

${ }^{2}$ West of Scotland Heart and Lung Centre, Golden Jubilee National Hospital, Glasgow, UK

${ }^{3}$ Advanced Cardiovascular Imaging Laboratory, National Heart, Lung, and Blood Institute, National Institutes of Health, Bethesda, Maryland, USA

${ }^{4}$ School of Biomedical Engineering and Imaging Sciences, Department of Cardiovascular Imaging, King's College London, London, UK

${ }^{5}$ Robertson Centre for Biostatistics, University of Glasgow, Glasgow, UK

Acknowledgements We thank the staff and patients who will support this study, and the BHF for financial support.

Contributors $\mathrm{CB}$ designed the study. $\mathrm{DC}$ wrote the first draft and revised draft with TJF, L-YH, AC, VO, KM, MM, PR, SW, RG, KB, SP, NS, AM, KGO, RT, AA and CB.

Funding This work was supported by the British Heart Foundation (BHF) (PG/17/2532884; RE/13/5/30177). The trial sponsor is the Golden Jubilee Research Foundation. The BHF has supported DC (FS/14/15/30661), TJF (RE/13/5/30177) and CB (RE/13/5/30177; FS/14/15/30661; FS172632744; PG-17- 25-32884).

Competing interests $\mathrm{CB}$ is employed by the University of Glasgow, which holds consultancy and research agreements with companies that have commercial interests in the diagnosis and treatment of angina. The companies include Abbott Vascular, AstraZeneca, Boehringer Ingelheim, Menarini Pharmaceuticals and Siemens Healthcare. KGO has received consultant and speaker fees from Abbott Vascular and Volcano Corporation which manufacture pressure wires. SW has worked as a consultant for Abbott Vascular. None of these companies have had any involvement with this study. None of the other authors have any potential conflicts of interest.

Patient consent for publication Not required.

Ethics approval The BHF CorMicA trial and prespecified CorCMR substudy has full UK National Research Ethics Service approval (Reference 16/WS/0192).

Provenance and peer review Not commissioned; externally peer reviewed.

Data sharing statement No additional data are available.

Open access This is an open access article distributed in accordance with the Creative Commons Attribution 4.0 Unported (CC BY 4.0) license, which permits others to copy, redistribute, remix, transform and build upon this work for any purpose, provided the original work is properly cited, a link to the licence is given, and indication of whether changes were made. See: http://creativecommons.org/ licenses/by/4.0

\section{REFERENCES}

1. GBD 2015 Mortality and Causes of Death Collaborators. Global, regional, and national life expectancy, all-cause mortality, and cause- 
specific mortality for 249 causes of death, 1980-2015: a systematic analysis for the Global Burden of Disease Study 2015. Lancet 2016;388:1459-544.

2. Excellence NlfHaC, 2010. Chest pain of recent onset: assessment and diagnosis. Available from: https://www.nice.org.uk/guidance/ cg95 [Accessed 20 Jun 2017].

3. Task Force M, Montalescot G, Sechtem U. ESC guidelines on the management of stable coronary artery disease: the task force on the management of stable coronary artery disease of the european society of cardiology. Eur Heart J 2013;34:2949-3003.

4. Patel MR, Peterson ED, Dai D, et al. Low diagnostic yield of elective coronary angiography. N Engl J Med 2010;362:886-95.

5. Ford TJ, Corcoran D, Berry C. Stable coronary syndromes: pathophysiology, diagnostic advances and therapeutic need. Heart 2018;104:284-92

6. Pries AR, Badimon L, Bugiardini R, et al. Coronary vascular regulation, remodelling, and collateralization: mechanisms and clinical implications on behalf of the working group on coronary pathophysiology and microcirculation. Eur Heart J 2015;36:3134-46.

7. Camici PG, Crea F. Coronary microvascular dysfunction. N Engl J Med 2007;356:830-40.

8. Lee JM, Jung JH, Hwang D, et al. Coronary flow reserve and microcirculatory resistance in patients with intermediate coronary stenosis. J Am Coll Cardiol 2016;67:1158-69.

9. Gulati M, Cooper-DeHoff RM, McClure C, et al. Adverse cardiovascular outcomes in women with nonobstructive coronary artery disease: a report from the women's ischemia syndrome evaluation study and the st james women take heart project. Arch Intern Med 2009;169:843-50.

10. Suwaidi JA, Hamasaki S, Higano ST, et al. Long-term follow-up of patients with mild coronary artery disease and endothelial dysfunction. Circulation 2000;101:948-54.

11. Murthy VL, Naya M, Taqueti VR, et al. Effects of sex on coronary microvascular dysfunction and cardiac outcomes. Circulation 2014;129:2518-27.

12. van de Hoef TP, van Lavieren MA, Damman P, et al. Physiological basis and long-term clinical outcome of discordance between fractional flow reserve and coronary flow velocity reserve in coronary stenoses of intermediate severity. Circ Cardiovasc Interv 2014;7:301-11.

13. Berry C. Stable coronary syndromes: the case for consolidating the nomenclature of stable ischemic heart disease. Circulation 2017:136:437-9.

14. Kirchhof P, Sipido KR, Cowie MR, et al. The continuum of personalized cardiovascular medicine: a position paper of the European Society of Cardiology. Eur Heart J 2014;35:3250-7.

15. Crea F, Lanza GA. Treatment of microvascular angina: the need for precision medicine. Eur Heart J 2016;37:1514-6.

16. Bairey Merz CN, Pepine CJ, Walsh MN, et al. Ischemia and No Obstructive Coronary Artery Disease (INOCA): developing evidencebased therapies and research agenda for the next decade. Circulation 2017;135:1075-92.

17. Ong P, Camici PG, Beltrame JF, et al. International standardization of diagnostic criteria for microvascular angina. Int $\mathrm{J}$ Cardiol 2018;250:16-20.

18. Galassi AR, Crea F, Araujo LI, et al. Comparison of regional myocardial blood flow in syndrome $\mathrm{X}$ and one-vessel coronary artery disease. Am J Cardiol 1993;72:134-9.

19. Meeder JG, Blanksma PK, Crijns $\mathrm{HJ}$, et al. Mechanisms of angina pectoris in syndrome $\mathrm{X}$ assessed by myocardial perfusion dynamics and heart rate variability. Eur Heart $J$ 1995;16:1571-7.

20. Camici PG, Crea F. Microvascular angina: a women's affair? Circ Cardiovasc Imaging 2015;8.

21. Hsu LY, Jacobs M, Benovoy M, et al. Diagnostic performance of fully automated pixel-wise quantitative myocardial perfusion imaging by cardiovascular magnetic resonance. JACC Cardiovasc Imaging 2018;11:697-707.

22. Schulz-Menger J, Bluemke DA, Bremerich J, et al. Standardized image interpretation and post processing in cardiovascular magnetic resonance: Society for Cardiovascular Magnetic Resonance (SCMR) board of trustees task force on standardized post processing. $J$ Cardiovasc Magn Reson 2013:15:35

23. Panza JA, Laurienzo JM, Curiel RV, et al. Investigation of the mechanism of chest pain in patients with angiographically normal coronary arteries using transesophageal dobutamine stress echocardiography. J Am Coll Cardiol 1997;29:293-301.

24. Bruder O, Wagner A, Lombardi M, et al. European Cardiovascular Magnetic Resonance (EuroCMR) registry--multi national results from 57 centers in 15 countries. J Cardiovasc Magn Reson 2013;15:9.

25. Marroquin OC, Holubkov R, Edmundowicz D, et al. Heterogeneity of microvascular dysfunction in women with chest pain not attributable to coronary artery disease: implications for clinical practice. Am Heart J 2003;145:628-35.

26. Panting JR, Gatehouse PD, Yang GZ, et al. Abnormal subendocardial perfusion in cardiac syndrome $\mathrm{X}$ detected by cardiovascular magnetic resonance imaging. $N$ Engl J Med 2002;346:1948-53.

27. Vermeltfoort IA, Bondarenko O, Raijmakers PG, et al. Is subendocardial ischaemia present in patients with chest pain and normal coronary angiograms? A cardiovascular MR study. Eur Heart J 2007;28:1554-8.

28. Karamitsos TD, Arnold JR, Pegg TJ, et al. Patients with syndrome $X$ have normal transmural myocardial perfusion and oxygenation: a 3-T cardiovascular magnetic resonance imaging study. Circ Cardiovasc Imaging 2012;5:194-200.

29. Thomson LE, Wei J, Agarwal M, et al. Cardiac magnetic resonance myocardial perfusion reserve index is reduced in women with coronary microvascular dysfunction. a national heart, lung, and blood institute-sponsored study from the women's ischemia syndrome evaluation. Circ Cardiovasc Imaging 2015;8.

30. Liu A, Wijesurendra RS, Liu JM, et al. Diagnosis of microvascular angina using cardiac magnetic resonance. J Am Coll Cardiol 2018;71:969-79.

31. Williams RP, de Waard GA, De Silva K, et al. Doppler versus thermodilution-derived coronary microvascular resistance to predict coronary microvascular dysfunction in patients with acute myocardial infarction or stable angina pectoris. Am J Cardiol 2018;121:1-8.

32. Ford TJ, Corcoran D, Oldroyd KG, et al. Rationale and design of the British heart foundation (bhf) coronary microvascular angina (cormica) stratified medicine clinical trial. Am Heart $J$ 2018;201:86-94.

33. Ford TJ, Rocchiccioli P, Good R, et al. Systemic microvascular dysfunction in microvascular and vasospastic angina. Eur Heart $J$ 2018.

34. Murthy VL, Naya M, Foster CR, et al. Association between coronary vascular dysfunction and cardiac mortality in patients with and without diabetes mellitus. Circulation 2012;126:1858-68.

35. Pepine CJ, Anderson RD, Sharaf BL, et al. Coronary microvascular reactivity to adenosine predicts adverse outcome in women evaluated for suspected ischemia results from the national heart, lung and blood institute wise (women's ischemia syndrome evaluation) study. J Am Coll Cardiol 2010;55:2825-32.

36. Taqueti VR, Hachamovitch R, Murthy VL, et al. Global coronary flow reserve is associated with adverse cardiovascular events independently of luminal angiographic severity and modifies the effect of early revascularization. Circulation 2015;131:19-27.

37. Fukushima K, Javadi MS, Higuchi T, et al. Prediction of short-term cardiovascular events using quantification of global myocardial flow reserve in patients referred for clinical 82Rb PET perfusion imaging. J Nucl Med 2011;52:726-32.

38. Serruys PW, di Mario C, Piek J, et al. Prognostic value of intracoronary flow velocity and diameter stenosis in assessing the short- and long-term outcomes of coronary balloon angioplasty: the debate study (doppler endpoints balloon angioplasty trial Europe). Circulation 1997;96:3369-77.

39. Chiribiri A, Villa AD, Sammut E, et al. Perfusion dyssynchrony analysis. Eur Heart J Cardiovasc Imaging 2016;17:1414-23.

40. Miller CA, Hsu LY, Ta A, et al. Quantitative pixel-wise measurement of myocardial blood flow: the impact of surface coil-related field inhomogeneity and a comparison of methods for its correction. $J$ Cardiovasc Magn Reson 2015;17:11

41. Benovoy M, Jacobs M, Cheriet F, et al. Robust universal nonrigid motion correction framework for first-pass cardiac MR perfusion imaging. J Magn Reson Imaging 2017;46:1060-72.

42. Zierler KL. Equations for measuring blood flow by externa monitoring of radioisotopes. Circ Res 1965;16:309-21.

43. Cerqueira MD, Weissman NJ, Dilsizian V, et al. Standardized myocardial segmentation and nomenclature for tomographic imaging of the heart. A statement for healthcare professionals from the cardiac imaging committee of the council on clinical cardiology of the American heart association. Circulation 2002;105:539-42.

44. Jerosch-Herold M, Wilke N, Stillman AE. Magnetic resonance quantification of the myocardial perfusion reserve with a Fermi function model for constrained deconvolution. Med Phys 1998;25:73-84

45. Axel L. Tissue mean transit time from dynamic computed tomography by a simple deconvolution technique. Invest Radiol 1983;18:94-9.

46. Hsu LY, Groves DW, Aletras AH, et al. A quantitative pixel-wise measurement of myocardial blood flow by contrast-enhanced first-pass CMR perfusion imaging: microsphere validation in 
dogs and feasibility study in humans. JACC Cardiovasc Imaging 2012;5:154-66.

47. Moon JC, Messroghli DR, Kellman P, et al. Myocardial t1 mapping and extracellular volume quantification: a Society for Cardiovascular Magnetic Resonance (scmr) and cmr working group of the european society of cardiology consensus statement. J Cardiovasc Magn Reson 2013;15:92

48. Kellman P, Arai AE, McVeigh ER, et al. Phase-sensitive inversion recovery for detecting myocardial infarction using gadoliniumdelayed hyperenhancement. Magn Reson Med 2002;47:372-83.

49. NICE, 2016. Chest pain of recent onset: assessment and diagnosis (NICE Guideline 95, updated November 2016). Available from: [https://www.nice.org.uk/guidance/cg95 [Accessed 5 Jan 2017].

50. Task Force Members, Montalescot G, Sechtem U. 2013 ESC guidelines on the management of stable coronary artery disease: the task force on the management of stable coronary artery disease of the european society of cardi ology. Eur Heart $J$ 20132013;34:2949-3003.
51. Plein S, Schwitter J, Suerder D, et al. k-Space and time sensitivity encoding-accelerated myocardial perfusion MR imaging at $3.0 \mathrm{~T}$ : comparison with 1.5 T. Radiology 2008;249:493-500.

52. Lockie T, Ishida M, Perera D, et al. High-resolution magnetic resonance myocardial perfusion imaging at 3.0-Tesla to detect hemodynamically significant coronary stenoses as determined by fractional flow reserve. J Am Coll Cardiol 2011;57:70-5.

53. Mygind ND, Michelsen MM, Pena A, et al. Coronary microvascular function and myocardial fibrosis in women with angina pectoris and no obstructive coronary artery disease: the iPOWER study. $J$ Cardiovasc Magn Reson 2016;18:76.

54. Wei J, Bakir M, Darounian N, et al. Myocardial scar is prevalent and associated with subclinical myocardial dysfunction in women with suspected ischemia but no obstructive coronary artery disease: from the women's ischemia syndrome evaluation-coronary vascular dysfunction study. Circulation 2018;137:874-6. 